$10-1993$

\title{
Order and Chaos in Semiconductor Microstructures
}

W. A. Lin

William \& Mary

John B. Delos

William \& Mary, jbdelos@wm.edu

Robert E. Jensen

Follow this and additional works at: https://scholarworks.wm.edu/aspubs

Part of the Physics Commons

\section{Recommended Citation}

Lin, W. A.; Delos, John B.; and Jensen, Robert E., Order and Chaos in Semiconductor Microstructures (1993). Chaos: An Interdisciplinary Journal of Nonlinear Science, 3(4), 655-664.

https://doi.org/10.1063/1.165994

This Article is brought to you for free and open access by the Arts and Sciences at W\&M ScholarWorks. It has been accepted for inclusion in Arts \& Sciences Articles by an authorized administrator of W\&M ScholarWorks. For more information, please contact scholarworks@wm.edu. 


\title{
Order and chaos in semiconductor microstructures
}

\author{
W. A. Lin and J. B. Delos \\ Institute for Theoretical Atomic and Molecular Physics, Harvard-Smithsonian Center for Astrophysics, \\ Cambridge, Massachusetts 02138 and Department of Physics, College of William \& Mary, \\ Williamsburg, Virginia 23185 \\ R. V. Jensen \\ Department of Physics, Texas A \& M University, College Station, Texas 77844
}

(Received 2 July 1993; accepted for publication 12 October 1993)

The semiclassical theory of ballistic electron transport in semiconductor microstructures
provides a description of the quantum conductance fluctuations in terms of the classical
distributions for the lengths and directed areas of the scattering trajectories. Because the
classical dynamics differs for integrable (circular) and chaotic (stadium) scattering domains,
experimental measurements of the conductance of these microstructures provide a unique probe
of the quantum properties of classically regular and chaotic systems. To advance these
theoretical and experimental studies we compare geometrical formulas for the classical
distributions of lengths and areas with numerical simulations for microstructures examined in
recent experiments, we assess the effects of lead size and placement, and we provide a critical
analysis of the role of scattering "noise" on the classical and semiclassical predictions. Finally,
we present a detailed comparison of the semiclassical theory with recent experimental
measurements of the conductance fluctuations in circular- and stadium-shaped microstructures.

\section{INTRODUCTION}

Advances in semiconductor fabrication techniques have produced solid-state devices that confine electrons to move in very small two-dimensional areas of the size of about a micron. Studies of the conduction properties of these "microstructures," which may someday form the components of very large scale integrated circuits, represent an exciting new area of research.

In high mobility GaAs microstructures the mean free path for elastic scattering of the electron from impurities is much larger than the size of the device. Consequently, the conducting electrons move freely through the device except at the confining walls where they undergo specular reflection. Moreover, at low temperatures $(\sim 20 \mathrm{mK})$ the mean free path for inelastic scattering is even longer and the electrons maintain their phase coherence for a long time inside the structure. As a result, the resistance of the device is primarily determined by the geometry of the structure.

In a recent experiment, ${ }^{1}$ the resistance across two different microstructures in the shape of a stadium and a circular disk was measured as a function of applied perpendicular magnetic field. Although the measured resistance was a complex function of the applied field in both cases, shape-dependent differences in the fluctuating resistance were clearly identifiable.

A detailed understanding of the complicated conductance fluctuations in these simple devices poses a challenge to theory. In addition to the practical considerations of the electrical properties of these devices, this experiment also provides a unique comparison of how regular and chaotic classical electron dynamics can affect the quantum transport. Since the classical motion of the ballistic electron in the stadium-shaped microstructure is chaotic while the classical dynamics in the circle is regular, a semiclassical description ${ }^{2}$ of the electron transport is required that can discriminate between regular and chaotic scattering of the conducting electrons as they traverse these devices.

In their seminal work, Jalabert, Baranger, and Stone ${ }^{3}$ combined the Landauer formula ${ }^{4}$ for the conductance,

$$
G=\frac{2 e^{2}}{h} \sum_{m, n}\left|t_{n m}\right|^{2},
$$

in terms of the complex transmission amplitudes $t_{n m}$ connecting the incoming and outgoing conduction channels, with Miller's semiclassical formula ${ }^{5}$ for the scattering amplitudes,

$$
t_{n m}=\sum_{r} a, e^{i \Phi_{r}}
$$

written as a weighted sum of the total semiclassical phases $e^{i \Phi_{r}}$ accumulated along each classical path $r$ connecting incoming channel $m$ with outgoing channel $n$, to derive a simple expression for the statistical correlation of the conductance fluctuations as a function of applied perpendicular magnetic field, $B$. The differences in the semiclassical phases are primarily determined by the magnetic flux passing through the areas, $A$, enclosed by the ballistic electron orbits as they pass through the device. Therefore, by replacing the weighted sums over classical orbits by integrals over the classical distributions of enclosed areas, the semiclassical correlation function for the conductance fluctuations can be simply expressed ${ }^{3}$ as,

$$
\langle\delta G(B+\Delta B) \delta G(B)\rangle \propto\left|\int_{-\infty}^{\infty} d A e^{i 2 \pi \Delta B A / \phi_{0}} P(A)\right|^{2},
$$

where $\phi_{0}=h / e=4.14 \times 10^{-3} \mathrm{~T} \mu \mathrm{m}^{2}$ is the fundamental unit of magnetic flux and $P(A)$ is the distribution of (directed) areas enclosed by the classical scattering trajectories. 
For weak magnetic fields the curvature of these classical electron orbits can be neglected and the areas computed using the straight line $(B=0)$ classical trajectories. Since numerical simulations ${ }^{3}$ indicated that these classical orbits through chaotic scattering domains exhibit an exponential decay in the distribution of directed areas,

$$
P(A) \propto e^{-\alpha|A|},
$$

the integral in Eq. (3) could be easily evaluated,

$$
\langle\delta G(B+\Delta B) \delta G(B)\rangle \propto 1 /\left[1+\left(2 \pi \Delta B / \alpha \phi_{0}\right)^{2}\right]^{2},
$$

to relate the semiclassical correlation of conductance fluctuations to the single classical exponent, $\alpha .^{6}$

In a previous paper, Jensen ${ }^{7}$ provided analytical derivations of the distributions of classical trajectories in various chaotic scattering domains with explicit geometrical formulas for the area decay exponents; $\alpha$, that can be conveniently used both to design and to interpret experiments of the ballistic electron transport in semiconductor microstructures. The primary purposes of the present work are to compare these analytical formulas with detailed numerical simulations of the classical scattering through twodimensional domains shaped like the chaotic stadium and regular circle and to use these analytical and numerical results to interpret recent experimental measurements which contrast the statistical properties of the conductance fluctuations in real microstructures with these shapes.

In Sec. II we describe the results of numerical simulations of the classical distributions of path lengths and directed areas for trajectories scattering through stadium and circle domains with two openings of variable width, $w$, corresponding to the experimental leads. For the chaotic stadium, the numerical distributions of classical lengths and areas are both exponential with characteristic exponents that are well described by the simple geometrical formulas of Ref. 7. In particular, we confirm in Sec. II A the predicted dependence of the area decay exponent on the square root of the width of the leads,

$$
\alpha_{s} \propto \sqrt{w}
$$

which has also been inferred in the recent analysis of experimental measurements of conductance fluctuations in stadium-shaped microstructures. ${ }^{8}$

In contrast, the numerical distributions for the path lengths and areas in the circle described in Sec. II B exhibit an initial exponential decay which crosses over to a power law behavior. Simple analytical arguments are presented to account for this behavior that can be generalized to any integrable scattering domain. In particular, we find that the initial exponential decay of the length distributions appears to be similar to the comparably sized stadium. However, because of the conservation of angular momentum in the integrable circle, the areas enclosed by the trajectories increase linearly with length and the initial exponential decay of the areas will be characterized by an exponent proportional to the lead widths,

$$
\alpha_{c} \propto w,
$$

which is very different from the chaotic case, Eq. (6). This new prediction may provide the clearest experimental means of distinguishing the regular and chaotic scattering domains.

In Sec. II C we briefly examine the dependence of the stadium results on the locations of the lead openings. Curiously, the classical distribution of lengths in the stadium can also exhibit a power law tail for some lead locations that lead to long-time trapping in the bouncing-ball motion between the two straight sides. However, because these orbits enclose very little directed area they cause no significant modifications to the exponential distribution of areas.

Some of the most important results of our numerical studies presented here are the illustrations in Sec. II D of the effects of random, nonspecular scattering that may arise from imperfections in real experimental microstructures. Here we find that strongly chaotic domains, like the long-sided stadium studied by Marcus et $a l .,{ }^{3}$ are relatively insensitive to this scattering "noise." However, less chaotic, short-sided stadium domains and the integrable circular domains are strongly affected by noise. In these cases the decay exponents for the lengths and areas are increased and the power law tails are destroyed. Nevertheless, the predicted linear dependence of the effective $\alpha_{c}$ for the circle on the lead widths, Eq. (7), appears to survive for small levels of nonspecular scattering.

Finally, in Sec. III the classical results for the area distributions, $P(A)$, for both the stadium and circle are used in combination with the semiclassical expressions for the correlation function, Eq. (3), to provide a detailed comparison of theory with recent experimental results. In particular, we show that the correlation of the measured conductance as a function of the magnetic field for the long-sided, chaotic stadium is well described by the classical area decay exponents, $\alpha_{s}$, evaluated for the specific devices used in the experiments. However, the experimental measurements for the circle cannot be adequately described using the theoretical results for the ideal circle. Only if we invoke large levels of nonspecular scattering noise in the classical simulations can we recover the fast (approximately) exponential decays observed in the experiment.

\section{CLASSICAL DYNAMICS}

\section{A. Chaotic billiard (stadium)}

For a billiard system with two holes (where particles enter from one of the holes), trajectories with different initial conditions spend different times inside the structure and travel different distances before escaping. Universal behavior associated with the underlying chaotic dynamics predicts that the probability density for traveling a distance $l$ then escaping is ${ }^{2} P(l) \propto e^{-\gamma l}$. In addition to this, for weak magnetic fields that do not significantly bend the straightline trajectories, one can also define an effective area $A \equiv(1 / B) \int \mathscr{A} \cdot d l$ accumulated along the straight-line path from entrance to escape where $\mathscr{A}$ is the vector po- 
tential for the applied magnetic field of strength $B$. Numerically, the probability density for acquiring an area $A$ before escaping is found to $\operatorname{be}^{3} P(A) \propto e^{-\alpha|A|}$.

Jensen ${ }^{7}$ showed, by means of the mixing property of a fully chaotic system, that the exponential decay exponent $\gamma$ can be related to geometrical dimensions of the microstructure,

$$
\gamma=\frac{\text { total size of holes }}{\pi(\text { area of container })} .
$$

For a stadium with two holes of equal size $w$, this gives

$$
\gamma=\frac{2 w}{\pi\left(\pi R^{2}+2 W R\right)}
$$

where $R$ is the radius of the semicircles and $W$ is the length of the straight sides. This formula is valid for small holes where the presence of the holes does not significantly affect the mixing property.

For various chaotic structures there is a general relationship ${ }^{7,9}$ between the two exponents $\gamma$ and $\alpha$,

$$
\alpha \propto \sqrt{\gamma} .
$$

The nontrivial dependence of $\alpha$ on the square root of $\gamma$ arises because the directed areas are accumulated in a random walk process as the scattered electron circulates one way and then the other as it bounces chaotically around the billiard. In particular, since $\gamma$ is proportional to the lead size, $w$, these results predict that the area decay exponent for the chaotic scattering domains will be proportional to the square root of the hole size, Eq. (6).

For a stadium billiard the geometrical formula ${ }^{7}$ gives,

$$
\alpha_{s} \simeq b \sqrt{6 R \gamma_{s}} / A_{s},
$$

where $A_{s}=\sqrt{2} R(\sqrt{2} R+W)$ is a typical magnitude for the enclosed area. For the strongly chaotic, long-sided stadium, $W>R$, the parameter $b$ was expected to be around 4 [Eq. (44) in Ref. 7]. However, because of the tendency for orbits to circulate several times around before reversing direction for the short-sided stadia with $W<R$, this parameter had to be reduced to $b \approx 1$ to achieve agreement with the numerical simulations by Jalabert $e t$ al. ${ }^{3}$ for shortsided stadia.

To perform our numerical simulations, we first adjust the length of the stadium and the size of the holes, then send in large number of particles (typically $10^{7}$ ) at different initial conditions, follow the orbits until they escape, and record $l$ and $A$. The distribution of the initial conditions is such that the particles are uniformly distributed over the entrance slit and at each position the directions of the particles are distributed uniformly over $\sin \beta$ where $\beta$ is the initial angle from the normal to the boundary at the entrance slit. If the resulting distributions of areas and lengths follow exponential laws, we fit the data to get $\gamma$ and $\alpha$.

In general, we find that if one of the leads is located on a curved side where the particles enter and the other located on a straight side, we get good exponential distribu-
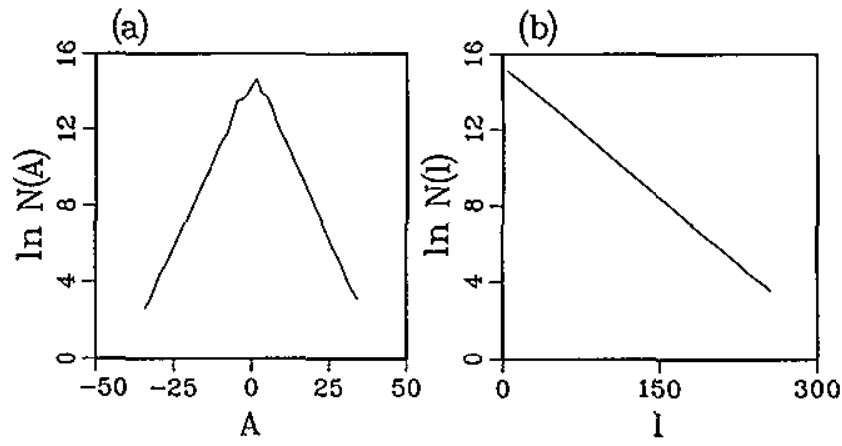

FIG. 1. Numerical simulations of the classical distributions for (a) directed areas, $N(A)$, and (b) lengths, $N(l)$, for the stadium microstructure of Marcus et al. ${ }^{1}$ We scale the lithographic dimension of the structure to correspond to $R=1, W=2$, and $w=0.4667$. (All the simulations done in this paper are for $R=1$.) One hole is centered on a semicircle, where $10^{7}$ particles are injected into the stadium, and the other is located at the joining point of the semicircle and one of the straight sides. For all the figures, $N(A)$ is the total number of particles that escaped and enclosed an area that is within the range of a bin centered at $A$, and similarly for $N(l)$. The best fit to the exponential decays of enclosed areas in (a) give $\alpha=0.351 \pm 0.003$, while Eq. (11) predicts $\alpha_{s}=0.414$, and the best fit to the exponential decay of lengths traveled in (b) gives $\gamma=0.0459$ \pm 0.0001 , while Eq. (9) predicts $\gamma_{s}=0.0416$.

tions that agree very well with the predictions of the simple geometrical formulas. For example, Fig. 1 shows the results for the length and area distributions for the stadium scattering domain with relative dimensions equivalent to the lithographic dimensions of the microstructures studied in Ref. 1. The exponential laws hold very well. In Fig. 2(a) we compare the numerical values of $\gamma$ as a function of side length $W$ with the analytical formula, Eq. (9), and Fig. 2 (b) verifies the linear relationship between $\alpha$ and $\sqrt{\gamma}$ predicted by Eq. (11).

For this long-sided stadium with $W / R=2$, the prediction of $\mathrm{Eq}$. (11) with $b \approx 4$ is in good agreement with the numerical simulations. However, for shorter-sided stadia Fig. 3 shows that the parameter, $b$, decreases approximately linearly with $W / R$ because of the increasing ten-
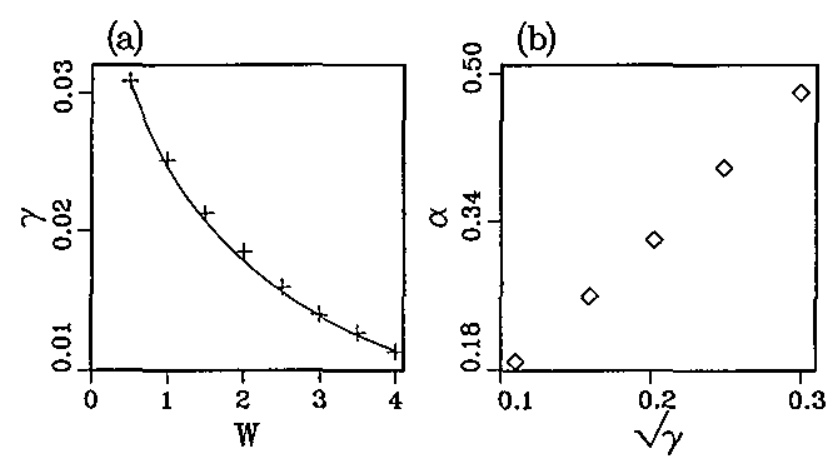

FIG. 2. Comparison of the numerical simulations with the geometrical formulas for the length and area decay exponents in the stadium. (a) Simulation results (plus) for $\gamma$ as a function of side length $W$ at fixed hole size $w=0.2$ give excellent agreement with Eq. (9) (solid curve). (b) For fixed side length, $W=4$, and different hole sizes, $w=0.2,0.4,0.6,0.8,1.0$, the simulation results (diamond) show a good linear relationship for $\alpha$ vs $\sqrt{\gamma}$ as predicted by Eq. (11). 


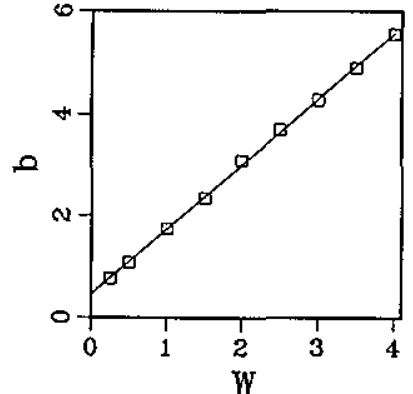

FIG. 3. The parameter $b$ in the geometrical formula for the area decay exponent, Eq. (11), increases linearly with stadium side length $W$. At fixed hole size, $w=0.2, \alpha$ is obtained from simulations at each side length, $W$, and the factor $b$ is extracted by making use of Eq. (11) and Eq. (9), The best fit line has a slope $1.28 \pm 0.01$ and $b$-intercept $0.45 \pm 0.03$.

dency for the orbits to circulate around the short-sided stadia. ${ }^{7}$ Figure 3 also shows that for longer-sided stadia the parameter $b$ continues to increase to values larger than 4 , presumably because the many orbits bouncing between the two straight sides enclose very little area.

\section{B. Integrable billiard (circle)}

Because of the conservation of angular momentum, the classical motion in the circular scattering domain is not chaotic. A scattering particle enters the circle with an initial angle relative to the boundary and preserves that angle at each bounce until it finds a hole and exits. In contrast with the irregular stadium dynamics, the particle orbits continue to circulate around the scattering domain in the same direction until they escape. Numerical simulations of the classical dynamics indicate that the distributions of lengths and areas exhibit an initial exponential decay which crosses over to a power law behavior for large path lengths or areas,

$$
\begin{aligned}
& P_{c}(l) \sim \begin{cases}e^{-\gamma_{c}}, & l \leqslant L_{c} ; \\
1 / l^{\beta_{l}}, & l>L_{c} ;\end{cases} \\
& P_{c}(A) \sim \begin{cases}e^{-\alpha_{c}|A|}, & A \leqslant A_{c} ; \\
1 / A^{\beta_{A}}, & A>A_{c} ;\end{cases}
\end{aligned}
$$

where $\beta_{l} \approx \beta_{A} \approx 3$ and $L_{c}$ and $A_{c}$ are the critical length and area where the distributions cross over from exponential to power law. For example, Fig. 4 shows the classical distributions of areas and lengths from our numerical simulations of scattering through an ideal circular domain corresponding to the lithographic dimensions of the circular device studied in Marcus et al.'s experiments. ${ }^{1}$

Our numerical simulations indicate that for small holes the initial exponential decay for the path lengths appears to be approximately described by the geometrical estimates for a chaotic billiard, Eq. (8), with the area of the circle

$$
\gamma_{c} \approx \frac{2 w}{\pi\left(\pi R^{2}\right)}
$$

(a)
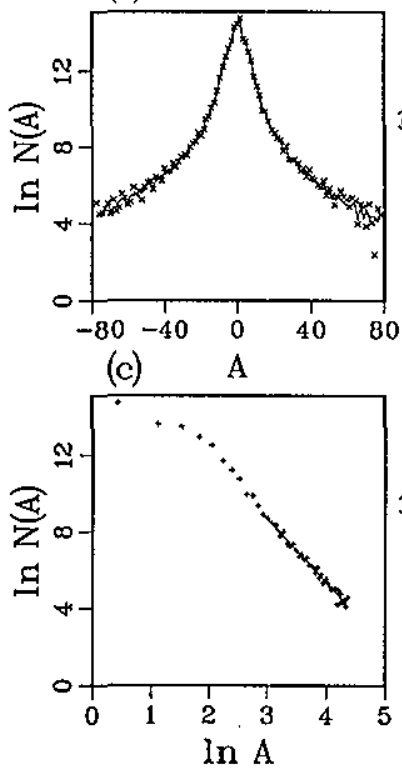

(b)

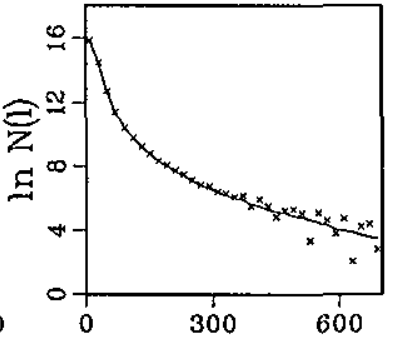

(d) 1

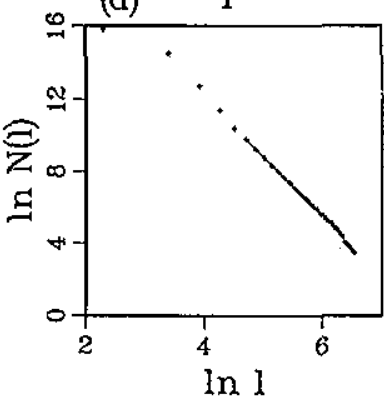

FIG. 4. Simulations of the area and length distributions for the circle of Marcus et $a l^{1}{ }^{1}$ using the lithographic dimensions scaled to $R=1$. The 2 holes of width $w=0.3182$ are separated by $90^{\circ}$. In (a) and (b), the $X$ symbols are for the case with the initial distribution of the particles at the entrance hole has 1000 positions and each position is given 10000 angles, while the line is for the case with 100 positions and 100000 angles. The distributions show exponential decays for small lengths or areas and long tails for larger lengths or areas. (c) The log-log plot shows that the long tail in the area distribution is a power law. A best fit line over the section shown has a slope $\beta_{A}=-3.33 \pm 0.07$. In (d) the log-log plot shows that the long tail in the length distribution is also a power law with slope $\beta_{l}=-3.26 \pm 0.02$.

This exponential decay can be partly understood by following the classical dynamics of the strip of initial conditions in the Birkoff phase space for the circular billiard with a single hole shown in Fig. 5. After $n$ bounces the linearly stretching strip of scattering trajectories will cross the loss strip $n$ times. Since the area of each intersection is proportional to the square of the width, $w$, times the slope, $\propto 1 / 2 \pi R n$, of the scattering strip, the total area of the $n$ intersections at the $n$th bounce will be $E_{n} \approx w^{2} / 2 \pi R$. If none of these orbits have escaped on previous bounces, then the fraction of orbits in the initial strip with phase space area $P(0)=2 w$ escaping on the $n$th bounce will be approximately $E_{n} / P(0)=w / 4 \pi R$ and the surviving probability after $n$ bounces will be

$P(n+1)=P(0)(1-n w / 4 \pi R) \approx P(0) e^{-(w / 4 \pi R) n}$,

for $n w / 4 \pi R \ll 1$.

Finally, assuming that a typical orbit traverses a distance of $\approx R$ between bounces, we arrive at a distribution of path lengths with an initial exponential decay of $\gamma=w / 4 \pi R^{2}$. If we double this rate for two holes of size $w$, this estimate is close to the chaotic value, Eq. (14).

Because of the regular dynamics in the circle, these arguments for the "average" behavior of "typical" orbits can only be expected to be qualitatively correct. The im- 
(a)

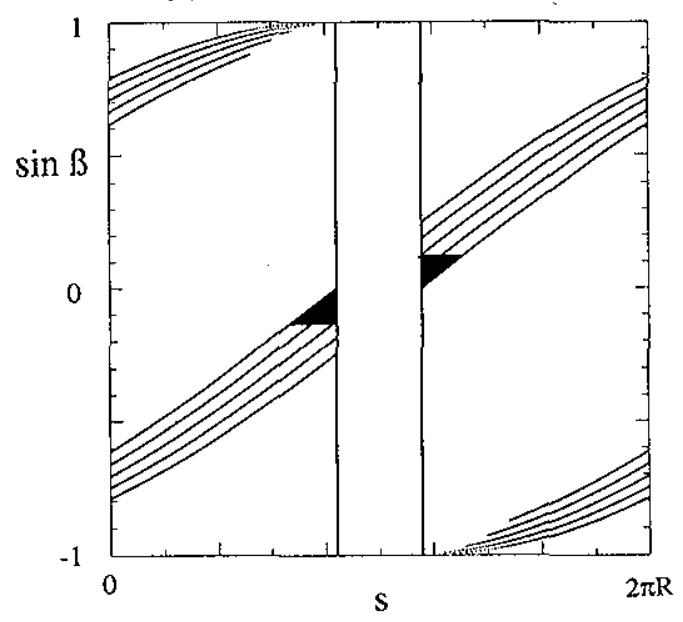

(b)

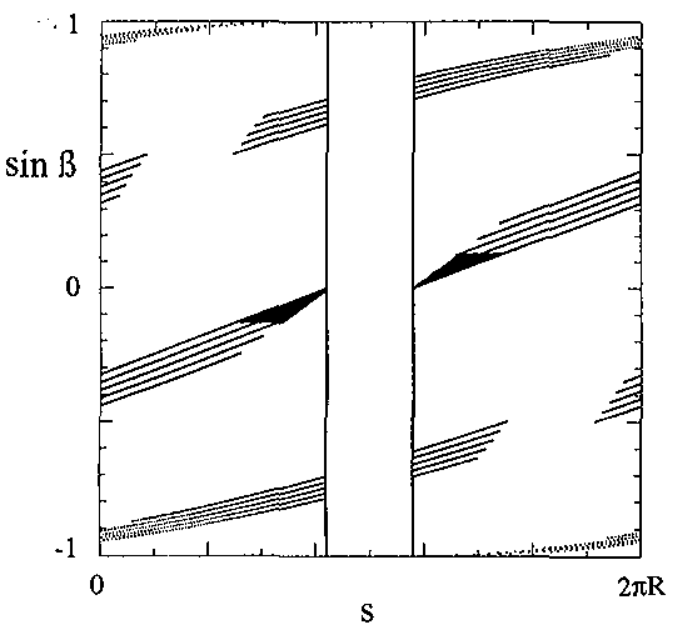

(c)

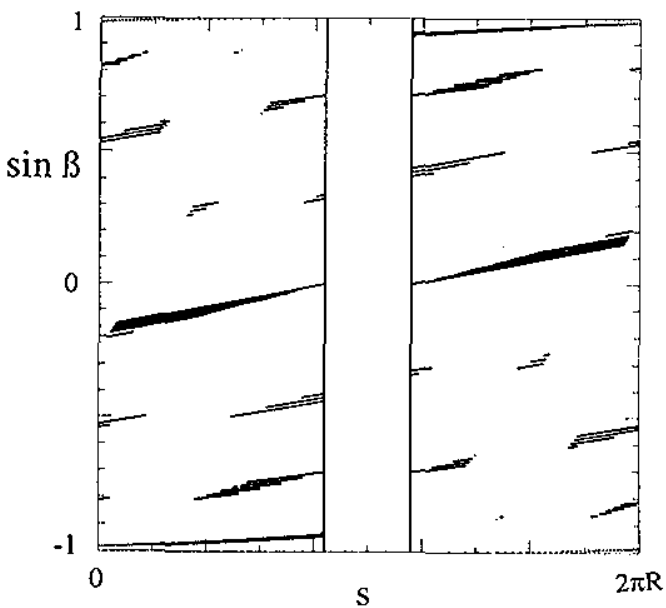

FIG. 5. The Birkoff phase space for a two-dimensional billiard system is a rectangle spanned by a coordinate variable, $s$, corresponding to the distance along the boundary for particle impact and a conjugate momentum variable given by $\sin \beta$ of the scattering angle $\beta$ relative to the normal direction from the boundary. The evolution of an initial strip of trajectories that entered a hole in the side of a circular domain, centered at $s=\pi R$ with a uniform distribution in $\sin \beta$, is shown. The time evolution in this integrable system causes the strip to stretch out at a linear rate and wrap around the rectangular phase space. This strip overlaps the vertical loss strip, corresponding to orbits that can exit the hole, in rhomboid-shaped regions. In (a) the initial strip is evolved for 2 bounces (indicated by the striped region) and the points representing the orbits escaping back through the opening are removed. In (b) the strip of evolving trajectories intersects the loss strip 4 times after 4 bounces, but two regions of overlap have already been depleted after 1 and 2 bounces. In addition, two gaps have appeared in the strip of trajectories that escaped on the third bounce. Finally, (c) shows the chopped up strip of orbits after 8 bounces. All three figures show two black, triangular regions that represent some of the longest lived trajectories that contribute to the long-time, power law tails in this integrable system. In (a) these triangles are shown with height $1 / 8$. After 4 bounces all of these orbits remain trapped in (b); however, after 8 bounces, some of these orbits have begun to escape and one corner of each triangle is chopped off. If the height of the triangle is reduced to $1 / 2 n$, these triangles of initial conditions with area $\propto 1 / n^{2}$ will survive $n$ bounces.

portant result is that these numerical simulations and analytical arguments indicate that the initial exponential decay rate for the circle should be proportional to the lead width as in the chaotic case. However, since the regular orbits in the circle always circulate in the same direction, the areas enclosed will also be proportional to the length (in contrast with the chaotic case) and the area decay exponents may be expected to scale linearly with lead width as in Eq. (7). In particular, the initial area decay exponent may be estimated by,

$$
\alpha_{c} \approx \gamma_{c} L / A \approx 4 \gamma_{c} / R
$$

where $L \approx 4 R$ is an estimate of a "typical" orbit length required to enclose an area $A \approx R^{2}$.

For $n \gg 4 \pi R / w$ the exponential decay predicted by Eq. (15) slows down because the escaping areas of phase space have already been depleted on previous bounces. (In contrast, the mixing property of the chaotic scattering domain ensures that the surviving orbits are uniformly mixed in phase space.) In the integrable system this steady decrease in the escape rate leads to an effective power law decay of the distributions of lengths and areas observed in the numerical simulations, Eqs. (12) and (13). Using the arguments above the crossover from exponential to power law 

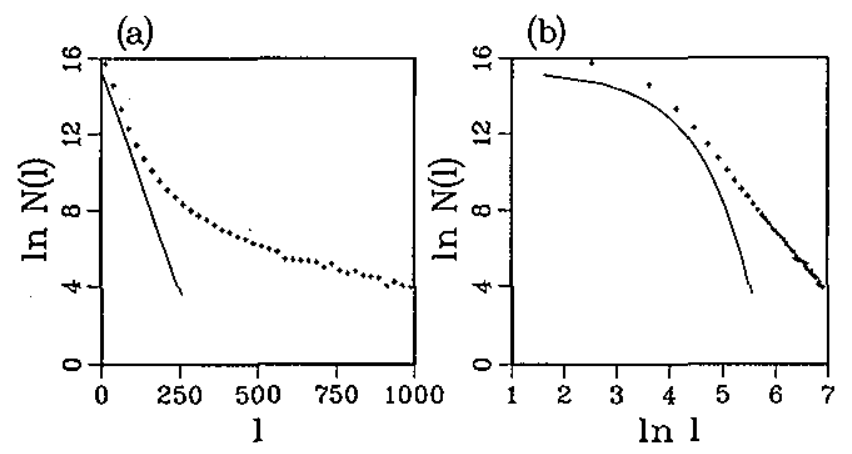

FIG. 6. This simulation for the area and length distributions of the stadium (+ symbols) differs from Fig. 1 (shown as lines in this figure) only in the location of the holes. In this case both holes are symetrically located on one of the curved ends. (a) The length distribution shows an initial exponential decay for small $l$ and a long tail for large $l$. The best line fit to the exponential part gives $\gamma=0.046 \pm 0.003$, which is basically the same as that in Fig. 1. (b) The log-log plot shows that the long tail is a power law. $A$ best fit line over the section shown has a slope $-3.18 \pm 0.06$. [The distribution of the areas is not significantly different from Fig. 1(a).]

can be roughly estimated to occur when the number of bounces exceeds $n_{c} \approx 4 \pi R / w$ and $l \gg L_{c} \approx 4 \pi R^{2} / w$ and $A \gg A_{c} \approx \pi R^{3} / w$.

The numerical simulations also indicate that this power law decay is characterized by a power around $\beta_{l} \simeq \beta_{A} \simeq 3$ (Ref. 10) which is different from the power of 2 for the escape of a uniform distribution of initial conditions in a regular domain through a single hole. ${ }^{11}$ Again a simple analytical argument can be invoked to account for this power of 3 . First, we note that the longest surviving orbits correspond to those initial conditions at the edge of the entrance slit that just miss escaping in a previous bounce. For example, the small triangles of initial conditions of height $\propto 1 / 2 n=1 / 8$ in Fig. 5(a) that just survived the first transit through the billiard will continue to remain completely trapped for $n=4$ bounces as shown in Fig. 5(b). However, this triangle of trapped orbits has already begun to erode after 8 bounces as indicated in Fig. 5(c). Since the phase space area of these triangles is $\propto 1 / 2 n^{2}$, the number of trajectories surviving longer than $n$ bounces represented by this region will be $N(n) \propto 1 / 2 n^{2}$ and the probability density for surviving $n$ bounces will be $P(n)=N^{\prime}(n) \propto 1 / n^{3}$.

\section{Location of holes}

We note that our numerical simulations do not always give exponential decays for the stadium. If we put both holes on the curved sides, we can obtain power law longtime tails for the distribution of lengths, as shown in Fig. 6. We have found ${ }^{12}$ that this comes about because a small fraction of particles enter into the region of phase space surrounding the family of periodic orbits bouncing between the two straight sides ${ }^{13}$ and they get trapped in that motion for a long time. The long-time tail begins to show up when most of the particles in the non-bouncing-ball region escape.
Once an orbit enters this bouncing-ball region it is hard to get out. The present situation is similar to a common trend for "nonhyperbolic" chaotic scattering, ${ }^{14}$ where a power law survival probability is obtained due to particles spending a long time near KAM surfaces. However, the stadium is not a near-integrable system and does not have KAM surfaces. So our present result shows a new possibility for the occurrence of a power law decay in a hyperbolic system.

\section{Effects of random scattering}

We next consider effects associated with deviations of real microstructures from the perfect circular and stadium billiard domains. In a real device the boundary may be rough rather than smooth; or inside the 2-D scattering domain the potential could be bumpy rather than flat. ${ }^{15}$ It would be hard to exactly simulate these imperfections. But the effect of these is to deflect the trajectory somewhat at each bounce from that of a perfect system. If we assume that these imperfections are distributed quite irregularly or randomly, then this situation can be simulated by the addition of a random angle in the range $[-a \pi, a \pi]$ to $\beta$ at each bounce, where $a \in[0,1]$ gives the amplitude of the noise. (If the addition of the random angle causes the reflected ray to go outside of the wall, we simply consider it as an incident ray and it gets reflected again by the perfect wall at the same position along the boundary.)

If the deviations from specular scattering in the ideal domain are due to irregularities in the boundary, the magnitude of the scattering "noise" measured by the parameter $a$ can be estimated from height $h$ and scale length $\lambda$ of the "ripple." For $h / \lambda \ll 1$, the scattering angle will deviate from the ideal value by an amount of order $h / \lambda$, so we can choose $a \approx h / \lambda$.

On the other hand if the deviations are due to potential bumps on the interior of the scattering domain, the deviation in the scattering angle can be estimated from the deflection of the trajectory as it traverses the domain. For potential bumps of height $V$ and scale length $\lambda$, the deflecting force will be proportional to $V / \lambda$. Since the force is only applied for a time, $t \approx 2 \lambda / v$, the transverse change in momentum due to the scattering from each bump can be estimated by $\Delta p / p \approx 2 V / m v^{2}$. In a solid state device this deflection of the scattering electron trajectory causes the scattering angle to change by an amount proportional to the ratio of the potential height, $V$, to the electron Fermi energy, $\epsilon_{f}=m v^{2} / 2$. If the electron traverses only a single bump in crossing the device then the magnitude of the scattering noise can be estimated by

$$
a \approx V / \epsilon_{f} .
$$

If the scale length of the bumps is much smaller than the size of the device, $\lambda \ll R$, then multiple scatterings can occur; however, for a random potential this scattering will be a random walk and the estimate for the noise amplitude, Eq. (17), will only be increased by a factor of $\approx \sqrt{R / \lambda}$.

Our numerical simulations indicate that the addition of random scattering noise has a small effect on the long-sided 
(a)

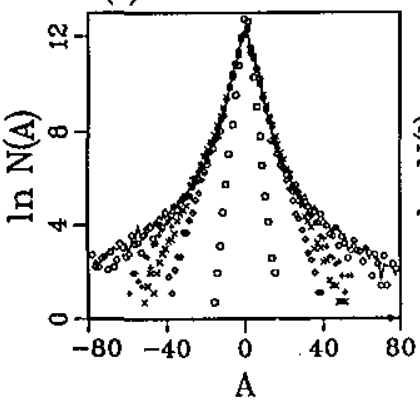

(b)

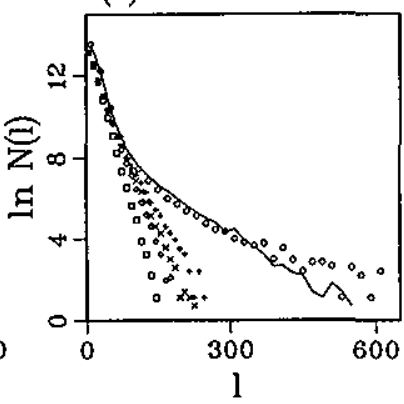

FIG. 7. The influence of scattering "noise" on the area and length distributions for the circular microstructure of Marcus et al. ${ }^{1}$ Different symbols are for different noise amplitudes: $a=0$ (circle), $a=0.001$ (line), $a=0.005$ (plus), $a=0.01$ ( $\times$ ), $a=0.05$ (diamond), and $a=0.5$ (square).

stadium with $W / R=2$. This is due to the inherent randomness of the chaotic motion in the stadium. The distributions remain good exponentials. An increase of $a$ from 0 to 0.5 only increases $\alpha_{s}$ from 0.35 to 0.46 and $\gamma_{s}$ from 0.046 to 0.054 . If we scale the results for $a=0.5$ to the lithographic dimensions of the stadium-shaped devices in Ref. 1 , we get $\alpha_{s}=5.1 \mu \mathrm{m}^{-2}$ and $\gamma_{s}=0.18 \mu \mathrm{m}^{-1}$.

For the circle, Fig. 7 shows that, at a small noise level of $a=0.001$, the distributions differ little from those for $a=0$. However, for $a=0.05$ we can already see a strong effect of the noise, which has turned most of the power law part into exponential. Beyond $a=0.05$, the distributions fit quite well with exponential decays and we see a large change in $\alpha_{c}$ going from 0.3 to 0.8 as $a$ is changed from 0.05 to 0.5 , while $\gamma_{c}$ has a modest change from 0.065 to 0.086 . Therefore, the random noise has a strong effect in reducing the ability of the particles to circulate. Scaled to the lithographic dimension of the circle in Ref. 1, these results for $a=0.5$ give $\alpha_{c}=4.1 \mu \mathrm{m}^{-2}$ and $\gamma_{c}=0.19 \mu \mathrm{m}^{-1}$. These are close to the exponents for the long-sided stadium. So we see that at high noise level it is hard to distinguish the circle from the stadium on the basis of the shape of the classical distributions of lengths and

(a)
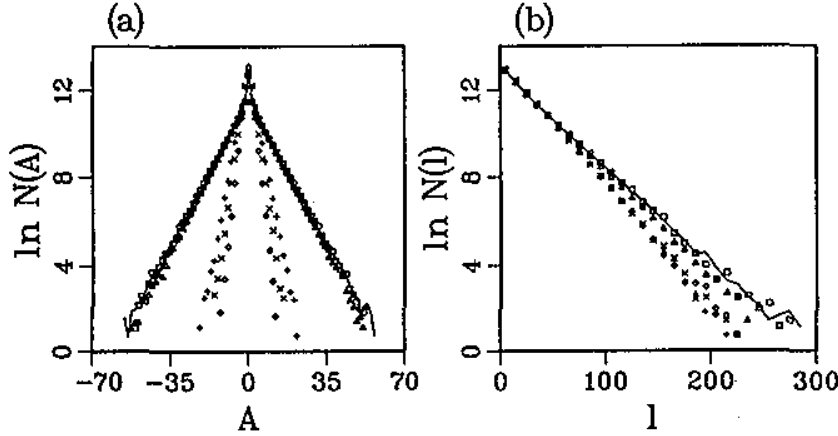

FIG. 8. The influence of scattering "noise" on the area and length distributions for the large stadium of Keller et al., ${ }^{17}$ scaled to $R=1, W$ $=0.2887, w=0.2887$. Different symbols are for different noise amplitudes: $a=0$ (square), $a=0.001$ (line), $a=0.05$ (triangle), $a=0.5$ (plus), $a=0.7$ $(X)$, and $a=1$ (diamond).

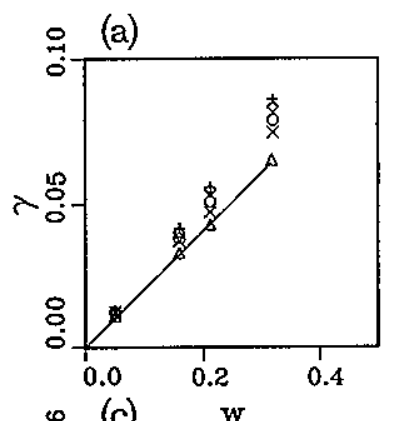

(b)
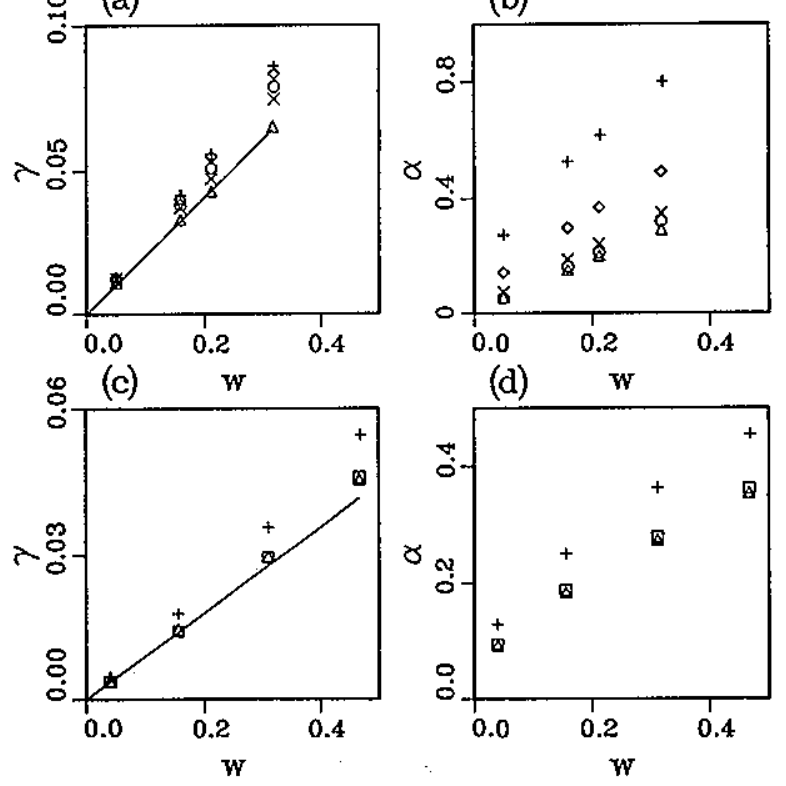

FIG. 9. Effects of noise on circles and stadium for different hole size $w$. In (a) and (b) the values of $\gamma$ and $\alpha$ for the exponential part of the distributions for the circle scale linearly with $w$ for zero or small levels of noise $a=0$ (circle), $a=0.05$ (triangle), and $a=0.1$ (cross). However, at the larger levels of noise, $a=0.25$ (diamond) and $a=0.5$ (plus), the dependence of $\alpha$ on $w$ begins to curve. The line in (a) shows the predictions of Eq. (14) for the initial exponential decay. In (c) and (d) the values of $\gamma$ and $\alpha$ for the stadium with $W=2$ at different hole sizes show the predicted dependence on $w$ and $\sqrt{w}$, respectively, for all noise levels, $a=0$ (square), $a=0.05$ (triangle), and $a=0.5$ (plus). The line in (c) shows the predictions of Eq. (9). Only for $w>0.4$ does the large hole size effect the ergodic property leading to significant deviations.

areas alone since the noise has destroyed the order in the circle that was responsible for the power law tails.

We also find that noise has a large effect on the tendency for orbits to circulate in the short-sided stadia. For example, Fig. 8 shows the numerical distributions of areas for different levels of nonspecular scattering for a shortsided stadium with $W / R=0.25$. In this case a noise level of $a=0.5$ increases the area decay exponent by as much as a factor of 3 .

Finally, we note that the significant differences in the scaling of the area decay exponent with hole size for the chaotic stadium, Eq. (6), and the regular circle, Eq. (7), appear to persist in the presence of small levels of scattering noise. As a consequence, this important distinction between the regular and chaotic scattering may survive in real measurements with "noisy" devices. For illustration, Fig. 9, shows the values of $\gamma$ and $\alpha$ as functions of hole size, $w$, for both the circle and the stadium with different levels of noise.

\section{COMPARISON OF THE SEMICLASSICAL THEORY AND EXPERIMENT}

The analytical and numerical results for the classical distributions of areas enclosed by particles scattering through the stadium and circle domains can now be incorporated into the semiclassical theory for the conductance 


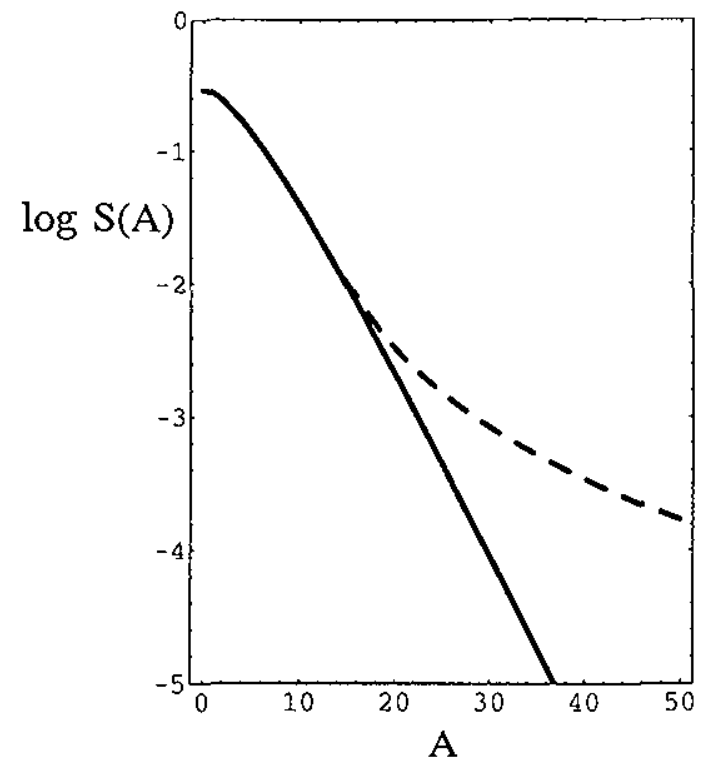

FIG. 10. The power spectrum predicted by the semiclassical theory, Eq. (18), for an exponential distribution of directed areas with $\alpha=0.35$ (solid curve) is compared with the results for the convolution of a distribution with an initial exponential decay with $\alpha=0.35$ that crosses over to a power law tail, Eq. (13), with $A_{c}=15$ (dashed curve). Here both convolutions of the respective area distributions are plotted in arbitrary units as functions of $A=\phi_{0} f$.

fluctuations for comparison with experimental measurements of the power spectrum of the conductance fluctuations. This is determined experimentally by the Fourier transform of the correlation function of conductance fluctuations measured as a function of magnetic field.

The semiclassical theory for weak magnetic fields, Eq. (3), expresses the correlation function of the conductance fluctuations as the square of the Fourier transform of the classical distribution of areas. Therefore, using the autocorrelation theorem, ${ }^{16}$ we can relate the theoretical prediction for the power spectrum of quantum conductance fiuctuations directly to a convolution of the classical area distributions,

$$
S\left(\phi_{0} f\right)=\int d A P(A) P\left(\phi_{0} f+A\right)
$$

as a function of the product of the magnetic "frequency," $f$ (in 1/Tesla) and the quantum unit of magnetic flux $\phi_{0}=h / e$.

In the case of the chaotic stadium, the exponential distribution of directed areas gives a simple prediction for the power spectrum ${ }^{1,3}$ of the conductance fluctuations,

$$
S\left(\phi_{0} f\right)=S(0)\left[1+\alpha \phi_{0} f\right] e^{-\alpha \phi_{0} f}
$$

in terms of the purely classical parameter $\alpha$. For an ideal circle the convolution of the exponential plus power law distribution, Eq. (13), cannot be expressed in a simple analytical form, but is similar to the exponential distribution for low magnetic frequencies and falls off less quickly than exponential for large areas or frequencies, $A=\phi_{0} f$ as shown in Fig. 10. However, as demonstrated in Sec. II D, the introduction of experimental "noise" can have a large effect on modifying the exponential decay of enclosed areas for short-sided stadia and for the circle and in suppressing the power law tail in the distributions for the circle. As a consequence, comparisons of ideal theory with realistic experiment must always keep in mind the potential influence of "noise."

By evaluating the classical distributions for the geometrical parameters of the experimental devices, these theoretical predictions can be directly compared with the experimental measurements. For example, in the experiments of Marcus et al. ${ }^{1}$ the conductance fluctuations have been measured as a function of applied magnetic field for microstructures shaped like a stadium and a circle. The lithographic dimensions of the stadium were $1.2 \mu \mathrm{m}$ in length and $0.6 \mu \mathrm{m}$ in width, corresponding to a radius of $R=0.3$ $\mu \mathrm{m}$ for the curved ends and a length of $W=0.6 \mu \mathrm{m}$ for the straight sides. The lithographic widths of the two attached leads, one at the center of one curved side and one at the near edge of the straight side, were $w=0.14 \mu \mathrm{m}$. The lithographic radius of the circle was chosen to be $R_{c}=0.44 \mu \mathrm{m}$ so the devices would have similar lithographic areas of $\approx 0.6 \mu \mathrm{m}^{2}$. Because of edge depletion the effective dimensions of the devices should be reduced. In fact, measurements ${ }^{1}$ in high magnetic fields of the AharonovBohm oscillations in the conductance due to magnetic flux enclosed by gyrating electrons circulating around the edge of the device indicates a reduction of the effective area by $30 \%$ corresponding to a decrease in the linear dimensions of the device and the leads of $15 \%$.

To compare with the experimental results, ${ }^{1}$ we define $\alpha^{\prime} \equiv \alpha / 2 \pi .^{6}$ The fits of Eq. (19) to the experimental data ${ }^{1}$ for the power spectrum gave values of $\alpha_{s}^{\prime-1}=1.2$ and 1.1 $\mu \mathrm{m}^{-2}$ for two stadium-shaped samples which correspond to values of $\alpha_{s}=5.2$ and $5.6 \mu \mathrm{m}^{-2}$. The value from our numerical simulations of the classical dynamics in the stadium using the lithographic dimensions of these devices is $\alpha_{s}=3.9 \mu \mathrm{m}^{-2}$ for the ideal stadium and $\alpha_{s}=5.1 \mu \mathrm{m}^{-2}$ for the noisy stadium with $a=0.5$ which are in good agreement with the experimental values. Even the simple geometrical formula for the area decay exponent, Eq. (11), in an ideal stadium gives a value of $\alpha_{s}=4.8 \mu \mathrm{m}^{-2}$ which is in remarkably good agreement with experiment. To the best of our knowledge, this provides a first successful comparison between theory and experiment for the conductance fluctuations in ballistic microstructures.

Marcus et al.'s experimental results ${ }^{1}$ for the power spectrum of the conductance fluctuations in the circular domain were very similar to those for the stadium at small "frequencies" but drop off more slowly for large values of $f$ corresponding to the survival of more orbits enclosing larger directed areas than in the stadium. Although the experimental results for the circle exhibit a qualitative resemblance to the theoretical curve for the convolution of the power law area distributions for the ideal circle shown in Fig. 10, quantitative agreement was not possible using either the lithographic or the $15 \%$ smaller effective dimensions of the circular microstructure. The theoretical predictions based on the numerical simulations of the classical 


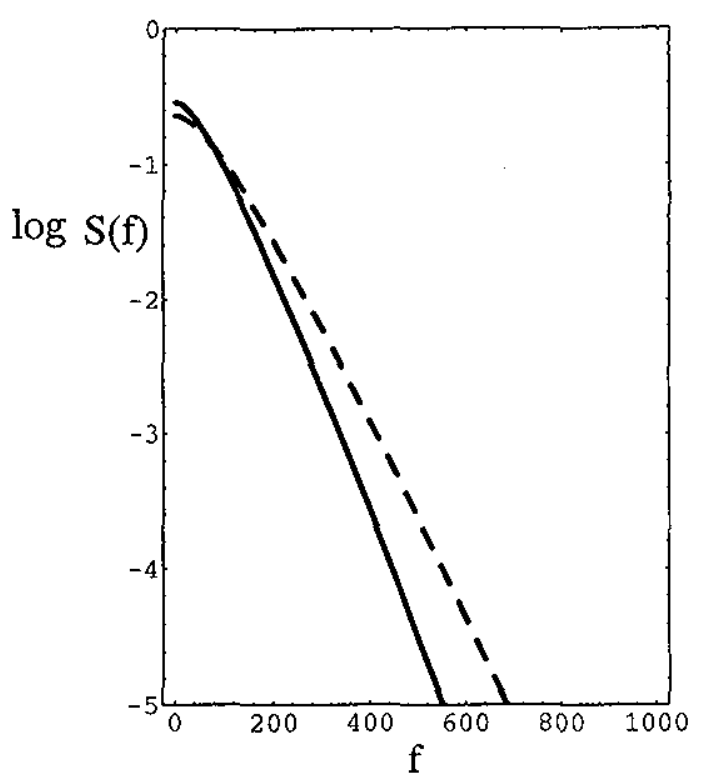

FIG. 11. The theoretical predictions for the power spectra for the noisy stadium (solid curve) and noisy circle (dashed curve) with noise level $a=0.5$ are plotted for the lithographic dimensions of the stadium- and circle-shaped microstructures studied in Ref. 1. The numerical simulations of the area distributions gave exponential distributions in both cases with $\alpha_{s} \simeq 5.1 \mu \mathrm{m}^{-2}$ for the stadium and $\alpha_{c} \approx 4.1 \mu \mathrm{m}^{-2}$ for the circle. The power spectra are plotted as a function of "magnetic" frequency $f$ measured in $1 /$ Tesla for direct comparison with the experimental results in Fig. 2 of Ref. 1.

scattering dynamics through the ideal circle give an initial value of $\alpha_{c} \simeq 1.7 \mu \mathrm{m}^{-2}$ which is approximately a factor of 2 or 3 slower decay for the exponential part the power spectrum than observed in the experiment.

However, as noted in our numerical studies of the effects of noise in Sec. II D, the addition of random scattering to the classical dynamics removes the power law tail from the distribution of areas for the circle and significantly increases the rate of decay while having little effect on the results for the long-sided stadium. Consequently, better quantitative agreement of theory and experiment can be achieved if we assume a large level of random, nonspecular scattering with $a \approx 0.5$ which gives a value of $\alpha_{c}=4.1 \mu \mathrm{m}^{-2}$ in much better agreement with the experiment. The predicted results of the semiclassical theory for the power spectra in both the stadium and circle with a noise level of $a=0.5$ are shown in Fig. 11 .

The addition of large amounts of scattering noise may also account for the apparent discrepancies between theory and experiment in the very recent experimental results by Keller et $a l .{ }^{17}$ for short-sided stadium-shaped microstructures. In Keller et al.'s experiment ${ }^{17}$ the conductance fluctuations of two short-sided stadia were studied, a large stadium with curved sides of radius, $R=0.6 \mu \mathrm{m}$, and straight side length, $W=0.15 \mu \mathrm{m}$, and a small stadium with $R=0.3 \mu \mathrm{m}$ and $W=0.15 \mu \mathrm{m}$. In both cases the lead widths spanned the entire straight side with $w=0.15 \mu \mathrm{m}$. Because of the tendency of the classical orbits to circulate in the same direction in the short-sided stadium, the theoretical predictions for $\alpha$ may be expected to be much
TABLE I. Comparison of experimental values of $\alpha$ (in $\mu \mathrm{m}^{-2}$ ) with numerical simulations using lithographic dimensions of the experimental structures with and without scattering noise.

\begin{tabular}{lccc}
\hline \hline Microstructure & Simulation $(a=0)$ & Simulation $(a=0.5)$ & Experiment \\
\hline $\begin{array}{l}\text { long stadium } \\
\text { circle }\end{array}$ & 3.9 & 5.1 & 5.2 \\
$\begin{array}{l}\text { short stadium } \\
\text { (large) }\end{array}$ & 1.7 & 4.1 & $\approx 4$ \\
$\begin{array}{l}\text { short stadium } \\
(\text { small) }\end{array}$ & 0.53 & 1.5 & 1.3 \\
\hline
\end{tabular}

smaller (with $b \approx 1$ ) for the short-sided stadium than for the long-sided stadium used in Marcus et al.'s experiments. ${ }^{1}$ But the experimental values ${ }^{17}$ for the decay of the conductance fluctuations indicated a value of $\alpha$ that was approximately a factor of 3 larger than theory. However, as shown in Fig. 8, the addition of noise has a large effect on the distribution of enclosed areas for the shortsided stadia that can account for the factor of 3. Consequently, this discrepancy may be reconciled if we again assume a large level of random, nonspecular scattering with $a \approx 0.5$.

The results of these comparisons of experimental measurements of the conductance fluctuations in long- and short-sided stadium- and circle-shaped microstructures with the semiclassical theory, including the effects of nonspecular scattering noise, are summarized in Table $\mathrm{I}$.

\section{ACKNOWLEDGMENTS}

We would like to thank C. M. Marcus, H. Baranger, M. W. Keller, E. J. Heller, J. D. Meiss, and M. J. Berry for helpful discussions, communications, and/or conversations. This research was supported by the Office of Naval Research and the National Science Foundation. It was also partially supported by the National Science Foundation through a grant for the Institute for Theoretical Atomic and Molecular Physics at Harvard University and Smithsonian Astrophysical Observatory.

${ }^{1}$ C. M. Marcus, A. J. Rimberg, R. M. Westervelt, P. F. Hopkins, and A. C. Gossard, Phys. Rev. Lett. 69, 506 (1992).

${ }^{2}$ R. Blümel and U. Smilansky, Phys. Rev. Lett. 60, 477 (1988) and references therein.

${ }^{3}$ R. A. Jalabert, H. U. Baranger, and A. D. Stone, Phys. Rev. Lett. 65, 2442 (1990).

${ }^{4}$ R. Landauer, IBM J. Res. Dev. 1, 223 (1957); M. Büttiker, IBM J. Res. Dev. 32, 317 (1988).

${ }^{5}$ W. H. Miller, Adv. Chem. Phys. 25, 69 (1974).

${ }^{6}$ In Refs. 1 and 3 the areas enclosed by the classical trajectories are perversely measured in units of $2 \pi$ times the physical area. As a consequence, their values of " $\alpha$ " are a factor of $2 \pi$ times smaller than our values for the area decay exponents measured for the true areas. Here we call their "area" decay exponent $\alpha^{\prime}$ which can be converted to our decay exponent by $\alpha \equiv 2 \pi \alpha^{\prime}$. This errant factor of $2 \pi$ can be the source of much confusion. [For example, in Eq. (16) of Ref. $7 \Delta B$ should be multiplied by $2 \pi$ or $\alpha$ changed to $\alpha^{\prime}$.]

${ }^{7}$ R. V. Jensen, Chaos 1, 101 (1991).

${ }^{8}$ C. M. Marcus, R. M. Westervelt, P. F. Hopkins, and A. C. Gossard, Phys. Rev. B15 48, 2460 (1993).

${ }^{9}$ E. Doron, U. Smilansky, and A. Frenkel, Physica D 50, 367 (1991).

${ }^{10}$ R. B. S. Oakeshott and A. MacKinnon, Superlat. Microstruct. 11, 145 (1992). 
${ }^{11}$ W. Bauer and G. F. Bertsch, Phys. Rev. Lett. 65, 2213 (1990).

${ }^{12}$ W. A. Lin, J. B. Delos, and R. V. Jensen (unpublished).

${ }^{13}$ F. Vivaldi, G. Casati, and I. Guarneri, Phys. Rev. Lett. 51, 727 (1983).

${ }^{14}$ Y.-C. Lai, R. Blümel, E. Ott, and C. Grebogi, Phys. Rev. Lett. 68, 3491

(1992) and references therein.

15 J. A. Nixon and J. H. Davies, Phys. Rev. B 41, 7929 (1990); J. A.
Nixon, J. H. Davies, and H. U. Baranger, Phys. Rev. B 43, 12638 (1991).

${ }^{16}$ R. Bracewell, The Fourier Transform and Its Applications (McGrawHill, New York, 1965), p. 115.

${ }^{17}$ M. W. Keller, O. Millo, A. Mittal, D. E. Prober, and R. N. Sacks, Surf. Sci. (in press, 1993). 